CALT-68-2251, CITUSC/99-008

HUTP-99/A070, LBNL-44695

UCB-PTH-99/54

hep-th/9912123

\title{
Knot Invariants and Topological Strings
}

\author{
Hirosi Oogurit团 and Cumrun Vafa* \\ $\dagger$ Caltech - USC Center for Theoretical Physics, Mail Stop 452-48 \\ California Institute of Technology, Pasadena, CA 91125 \\ * Jefferson Laboratory of Physics, Harvard University, Cambridge, MA 02138 \\ hooguri@lbl.gov, vafa@string.harvard.edu
}

\begin{abstract}
We find further evidence for the conjecture relating large $N$ Chern-Simons theory on $S^{3}$ with topological string on the resolved conifold geometry by showing that the Wilson loop observable of a simple knot on $S^{3}$ (for any representation) agrees to all orders in $N$ with the corresponding quantity on the topological string side. For a general knot, we find a reformulation of the knot invariant in terms of new integral invariants, which capture the spectrum (and spin) of M2 branes ending on M5 branes embedded in the resolved conifold geometry. We also find an intriguing link between knot invariants and superpotential terms generated by worldsheet instantons in $N=1$ supersymmetric theories in 4 dimensions.
\end{abstract}

December 1999

1 On leave of absence from University of California, Berkeley. 


\section{Introduction}

Large $N$ gauge theories have been conjectured by 't Hooft to be related to string theories. A particularly simple example of gauge theories is the Chern-Simons theory, solved by Witten. It is thus natural to ask about the large $N$ limit of Chern-Simons theory and look for an appropriate stringy description. Some aspects of large $N$ limit of Chern-Simons theory were studied some time ago in [1], [2].

It was conjectured recently [3] that at least for some manifolds (including $S^{3}$ ) the large $N$ limit does give rise to a topological string theory on a particular Calabi-Yau background. This conjecture was checked at the level of the partition function on both sides; The ChernSimons answer was already well known, and the topological string partition function was recently computed in two different ways (one by mathematicians, and one by using physical reasoning about the structure of BPS states).

It is natural to extend the conjecture to the observables of Chern-Simons theory, which are Wilson loop operators. Namely we should consider product of Wilson loop observables for any choice of representation on each knot. We show how this question can be formulated in the present context and explicitly check the map for the case of the simple circle in $S^{3}$ ("unknot"). The computation on the Chern-Simons side is well known. On the topological string side, we end up with topological string amplitudes on Riemann surfaces with boundaries. Mathematically these have not been studied, however by connecting the partition function of topological strings to target space quantities we compute them in terms of spectrum of M2 branes ending on M5 branes embedded in the Calabi-Yau threefold. The target space interpretation is also related to generation of $N=1$ superpotential terms in four dimensions (which we relate it analogously to the spectrum of domain walls).

For a general knot finding the explicit form of the M5 brane embedded in the CalabiYau is not trivial, though physically we argue it should be possible. In this way we reformulate knot invariants in terms of new invariants capturing the spectrum of M2 branes ending on M5 branes.

The organization of this paper is as follows: In section 2 we review the large $N$

conjecture for Chern-Simons theory. In section 3 we show how the Wilson Loop observable for arbitrary knot and representation can be formulated in this set up, and apply the gauge theory/geometry correspondence for the case of the simple knot. In section 4 we show how the results anticipated from the Wilson loop observables can be directly obtained using 
the spectrum of M2 branes ending on M5 branes (or D2 branes ending on D4 branes). We also point out connections with generation of superpotential terms with theories with 4 supercharges. In section 5 we present some concluding remarks and suggestions for future work.

\section{The Large $N$ conjecture for Chern-Simons Theory}

In this section we review the conjecture of [3] which relates large $N$ limit of $S U(N)$ Chern-Simons gauge theory on $S^{3}$ to a particular topological string amplitude. The motivation for the conjecture was that, in the context of topological strings of the $A$-type on Calabi-Yau threefolds, there are D-branes with three-dimensional worldvolume which support the Chern-Simons gauge theory [4]. So it is natural to expect that at least in some cases, by putting many branes on some cycles and taking the large $N$ limit, we end up with a topological string on some deformed Calabi-Yau, but without branes. This is what was found to be the case in [3], which we will now review.

\subsection{The statement of the conjecture}

The conjecture in [3] states that the Chern-Simons gauge theory on $S^{3}$ with gauge group $S U(N)$ and level $k$ is equivalent to the closed topological string theory of $A$-type on the $S^{2}$ blown up conifold geometry with

$$
\lambda=\frac{2 \pi}{k+N}, \quad t=\frac{2 \pi i N}{k+N},
$$

where $\lambda$ is the string coupling constant and $t$ is the Kähler modulus of the blown-up $S^{2}$. The coupling constant $g_{C S}$ of the Chern-Simons theory, after taking into account the finite renormalization, is related to $\lambda$ as $\lambda=g_{C S}^{2}$. Therefore the Kähler moduli $t$

given by (2.1) is $i$ times the 't Hooft coupling $g_{C S}^{2} N$ of the Chern-Simons theory. The geometric motivation of the conjecture is based on starting with the topological strings on conifold geometry $T^{*} S^{3}$ and putting many branes on $S^{3}$, for which we get a large $N$ limit of Chern-Simons on $S^{3}$ supported on the brane. The conjecture states that in the large $N$ limit the branes disappear but lead instead to the resolution of the conifold geometry where an $S^{2}$ has blown up. In fact this conjecture parallels the motivation for the AdS/CFT correspondence conjecture: As noted in [3], since the open topological string theory couples to closed topological string theory through a gravitational Chern-Simons 
action [4], putting 3-branes on $S^{3}$ deforms the gravitational background so as to produce a blown up $S^{2}$. In fact the volume of the $S^{2}$ was computed in this way.

The conjecture has been checked as follows: Start with the vacuum amplitude $Z\left(S^{3}\right)$ of the Chern-Simons gauge theory on $S^{3}$ (with the normalization $Z\left(S^{2} \times S^{1}\right)=1$ );

$$
Z\left(S^{3}\right)=\frac{e^{i \frac{\pi}{8}(N-1) N}}{(k+N)^{N / 2}} \sqrt{\frac{k+N}{N}} \prod_{s=1}^{N-1}\left[2 \sin \left(\frac{s \pi}{k+N}\right)\right]^{N-s} .
$$

The large- $N$ expansion of $\log Z\left(S^{3}\right)$ is given by

$$
Z\left(S^{3}\right)=\exp \left[-\sum_{g=0}^{\infty} \lambda^{2 g-2} F_{g}(t)\right]
$$

where $\lambda$ and $t$ as in (2.1),

$$
\begin{aligned}
& F_{0}=-\zeta(3)+\frac{i \pi^{2}}{6} t-i\left(m+\frac{1}{4}\right) \pi t^{2}+\frac{i}{12} t^{3}+\sum_{n=1}^{\infty} n^{-3} e^{-n t} \\
& F_{1}=\frac{1}{24} t+\frac{1}{12} \log \left(1-e^{-t}\right)
\end{aligned}
$$

with $m$ being some integer, and for $g \geq 2$,

$$
F_{g}=\frac{(-1)^{g-1}}{2 g(2 g-2)} B_{g}\left[\frac{(-1)^{g-1}}{(2 \pi)^{2 g-2}} 2 \zeta(2 g-2)-\frac{1}{(2 g-3) !} \sum_{n=1}^{\infty} n^{2 g-3} e^{-n t}\right] .
$$

Here $B_{g}$ is the Bernoulli number, which is related to the Euler characteristic of the moduli space $\mathcal{M}_{g}$ of genus- $g$ Riemann surfaces as

$$
\chi_{g}=\frac{(-1)^{g-1}}{2 g(2 g-2)} B_{g} .
$$

By using this and the formula for the Chern-class of the Hodge bundle over the moduli space

$$
\int_{\mathcal{M}_{g}} c_{g-1}^{3}=\frac{(-1)^{g-1}}{(2 \pi)^{2 g-2}} 2 \zeta(2 g-2) \chi_{g},
$$

which was derived in [5], one can rewrite (2.5) as

$$
F_{g}=\int_{\mathcal{M}_{g}} c_{g-1}^{3}-\frac{\chi_{g}}{(2 g-3) !} \sum_{n=1}^{\infty} n^{2 g-3} e^{-n t} .
$$

It turns out that the expressions (2.4) and (2.8) for $F_{g}$ are exactly those of the $g$-loop topological string amplitude on the resolved conifold. The constant map from the worldsheet 
to the target space gives rise to the term $\frac{i}{12} t^{3}$ in $F_{0}$ [6] 2 , and $\frac{1}{24} t$ in $F_{1}$ [8], and $\int_{\mathcal{M}_{g}} c_{g-1}^{3}$ in $F_{g}$ with $g \geq 2$ [9]. Regarding worldsheet instantons, since the only non-trivial 2-cycle in the target space is the blown-up $S^{2}$, their contributions are from multi-coverings of the Riemann surface onto the $S^{2}$. For $g=0,1$ and 2, the expressions in the instanton terms in (2.4) and (2.5) agree with the results of [6], [8], and [9] respectively. More recently, the contribution of of the worldsheet instantons are evaluated for all $g$ in [5], in complete agreement with (2.8). These expressions can also be derived, as was done in [10], from the target space view point by identifying what the topological strings compute in Type IIA compactification on the corresponding Calabi-Yau threefold. It turned out that the full structure of $F_{g}$ is encoded in terms of the spectrum of wrapped D2 branes on the Calabi-Yau. This will be reviewed later in this paper.

In this paper, we provide further evidence for the conjecture. We will show that the Wilson loop expectation value of the Chern-Simons theory also has a natural interpretation in terms of the topological string on the resolved conifold geometry.

\subsection{Conifold transition}

As noted above, the geometric insight that led to the conjecture is the fact that one can view the Chern-Simons theory as the open topological string theory. Consider the cotangent space $T^{*} S^{3}$ of $S^{3}$ as the target space of the topological string. It was shown in [4] that, if we wrap $N$ D-brane on the base $S^{3}$ of the cotangent space, the open topological string theory on the D-brane is equivalent to the Chern-Simons theory with the gauge group $S U(N)$. The cotangent space has the canonical symplectic form

$$
\omega=\sum_{i=1}^{3} d q^{i} \wedge d p_{i}, \quad\left(q \in S^{3}, p \in T_{q} S^{3}\right),
$$

and the base $S^{3}$ is a Lagrangian submanifold. Therefore the open string on the D-brane allows the topological twist of the $A$-type.

At this point, it would be useful to review basic facts about the conifold transition. The space $T^{*} S^{3}$ can also be regarded as a deformed conifold geometry,

$$
\sum_{\mu=1}^{4} y_{\mu}^{2}=a^{2}, \quad\left(y \in C^{4}\right)
$$

2 The coefficients of 1 and $t$ in $F_{0}$ have analogous interpretations [7], and they also agree with the Chern-Simons prediction [3]. 
where, without loss of generality, we assume the deformation parameter $a$ to be real. To see that $(2.10)$ is indeed $T^{*} S^{3}$, we can set $y_{\mu}=x^{\mu}+i p_{\mu}$ and rewrite the equation as

$$
\left(x^{\mu}\right)^{2}-\left(p_{\mu}\right)^{2}=a^{2}, \quad x^{\mu} p_{\mu}=0 .
$$

The first equation suggests that the base $S^{3}$ of radius $a$ is located at $p_{\mu}=0$ and the second equation shows that $p_{\mu}$ are coordinates on the cotangent space at $x \in S^{3}$. As $a \rightarrow 0$, the $S^{3}$ shrinks to a point and a singularity appears. It is known as the conifold singularity.

In addition to the deformation by $a$, the conifold singularity

$$
\sum_{\mu} y_{\mu}^{2}=0
$$

can be smoothened out by the blow-up. It is described as follows. By introducing two pair of complex coordinates $(u, \tilde{u})$ and $(v, \tilde{v})$ by

$$
\begin{array}{ll}
u=y_{1}+i y_{2}, & \tilde{u}=y_{3}-i y_{4} \\
v=y_{3}+i y_{4}, & \tilde{v}=y_{1}-i y_{2},
\end{array}
$$

the equation (2.12) can be written as

$$
u \tilde{v}+v \tilde{u}=0 .
$$

This means that there is some $z$ such that

$$
u=z \tilde{u}, \quad v=-z \tilde{v} .
$$

If we view $z$ as a complex coordinate on $S^{2}$ (as we should since $\tilde{u}$ can be 0 and we need to add $z=\infty)$, one can interpret $(2.15)$ as defining the bundle $\mathcal{O}(-1)+\mathcal{O}(-1)$ over $S^{2}$ where $u$ and $v$ are coordinates on the fibers. With respect to the original symplectic form (2.9), the volume of $S^{2}$ is zero, which is another way to see that the conifold geometry is singular. We can remove the singularity by blowing up the $S^{2}$; this process is called the small resolution (as opposed to the deformation of complex structure $a$ in the previous paragraph). See Figure 1. The conifold singularity (2.14) can be either deformed to the total space of $T^{*} S^{3}$ or resolved to the total space of $\mathcal{O}(-1)+\mathcal{O}(-1)$ over $S^{2}$. The transition from one to the other is called the conifold transition.

The conjecture in [3] states that the open topological string theory on the $N$ D-branes on $S^{3}$ of the deformed conifold is equivalent to the closed topological string theory (without D-branes) on the resolved conifold with $t=2 \pi i N /(k+N)$. 


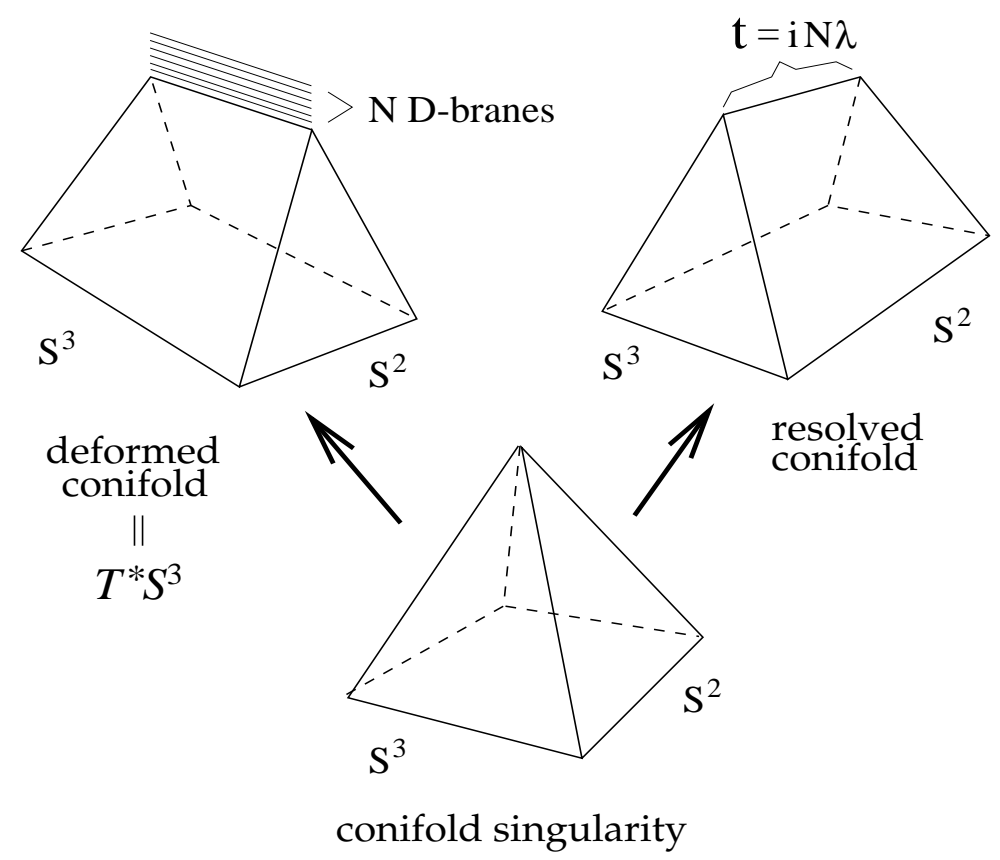

Figure 1: The conifold singularity can be either deformed to $T^{*} S^{3}$ or resolved by the $S^{2}$ blow-up. The conjecture in [3] states that the open topological string theory on the $N$ D-branes on $S^{3}$ of the deformed conifold is equivalent to the closed topological string theory on the resolved conifold geometry.

\section{Wilson loop}

\subsection{Definition}

The basic observables of the Chern-Simons gauge theory are the Wilson loops. For each loop $q(s) \in S^{3}(0 \leq s<2 \pi)$, we can define a generating function of Wilson loops of various representations of $S U(N)$ in the following way. As shown in [प], the Chern-Simons theory is the open topological string theory on $N$ D-branes wrapping the base $S^{3}$ of $T^{*} S^{3}$. We can probe the dynamics on these D-branes by introducing another set of D-branes. First we define a Lagrangian 3-cycle associated to the knot $q(s) \in S^{3}$ as follows 3 . At each point $q(s)$ on the loop, we consider 2-dimensional subspace of $T_{q}^{*} S^{3}$ orthogonal to $d q / d s$. By going around the loop, we can define the 3-cycle,

$$
\mathcal{C}=\left\{(q(s), p) \mid p_{i} \frac{d q^{i}}{d s}=0, \quad 0 \leq s<2 \pi\right\} .
$$

3 We thank C. Taubes for discussion on these Lagrangian cycles. 
The topology of $\mathcal{C}$ is $R^{2} \times S^{1}$. The symplectic form $\omega$ vanishes on $\mathcal{C}$, so it is a Lagrangian submanifold目. The 3 -cycle intersects with the base $S^{3}$ along the loop $q(s)$. See Figure 2 .

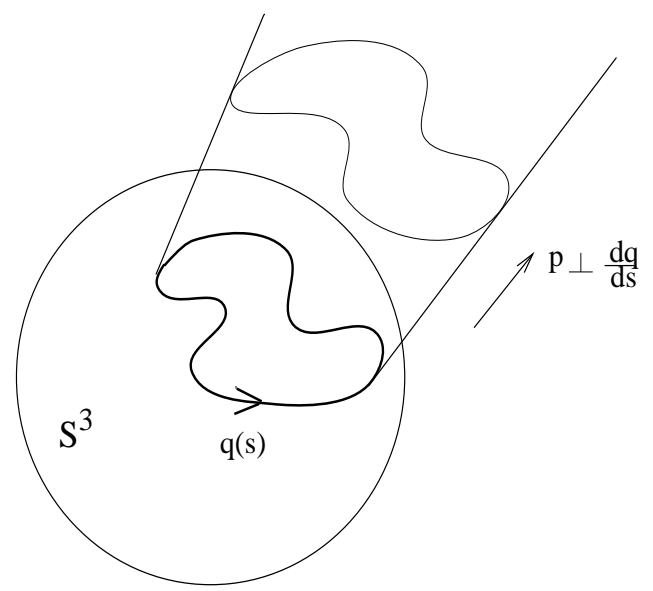

Figure 2: For each loop $q(s) \in S^{3}$, one can define a unique Lagrangian 3-cycle which extends in the cotangent direction and intersects $S^{3}$ on the loop $q(s)$.

Now let us wrap $M$ D-branes on $\mathcal{C}$. We then have the $S U(M)$ Chern-Simons theory on $\mathcal{C}$ as well as the $S U(N)$ Chern-Simons theory on $S^{3}$. In addition, we also have a new sector of open string with one end on $\mathcal{C}$ and the other on $S^{3}$. One can easily quantize the topological string in this sector and obtain a complex scalar field living on the intersection, namely the loop $q(s)$, which transforms according to the bi-fundamental of $S U(N) \otimes$ $S U(M)$. To see that there is one complex scalar field of this type, we note that, in the relevant open string sector i.e. the Ramond sector, there are two states one with $N=2$ $U(1)$ charge $-1 / 2$ (a scalar) and the other with $+1 / 2$ (a 1-form). The physical states of the topological string come from the sector with $U(1)$ charge $-1 / 2$, and that turns out to correspond to the scalar living on the loop $q(s)$. The action for the scalar field is Gaussian, and integrating it out gives the determinant,

$$
Z=\exp \left[-\log \operatorname{det}\left(\frac{d}{d s}+\left(A_{i}-\tilde{A}_{i}\right) \frac{d q^{i}}{d s}\right)\right],
$$

where $A$ and $\tilde{A}$ are the Chern-Simons gauge fields on $S^{3}$ and $\mathcal{C}$ respectively. We can evaluate the determinant by diagonalizing $A$ and $\tilde{A}$ (this is allowed since we are dealing with the one-dimensional problem along the intersection). By using the formula

$$
\log \operatorname{det}\left[\frac{d}{d s}+i \theta\right]=\sum_{n=-\infty}^{\infty} \log (n+\theta)=\log \sin (\pi \theta)+\text { const }
$$

4 The cycle $\mathcal{C}$ defined here is Lagrangian but is not necessarily special Lagrangian. In order for to make the topological $A$-twist, it is sufficient that $\omega$ vanishes on $\mathcal{C}$. 
we find

$$
\begin{aligned}
Z(U, V) & =\exp \left[-\operatorname{tr} \log \left(U^{-1 / 2} \otimes V^{1 / 2}-U^{1 / 2} \otimes V^{-1 / 2}\right)\right] \\
& =\exp \left[-\operatorname{tr} \log \left(1-U \otimes V^{-1}\right)\right] \\
& =\exp \left[\sum_{n=1}^{\infty} \frac{1}{n} \operatorname{tr} U^{n} \operatorname{tr} V^{-n}\right]
\end{aligned}
$$

where $U$ and $V$ are path-ordered exponentials of the gauge fields along the loop,

$$
U=P \exp \oint A \in S U(N), \quad V=P \exp \oint \tilde{A} \in S U(M)
$$

and we used $\operatorname{det} U=\operatorname{det} V=1$.

We are interested in taking $N$ to infinity for a fixed $M$. We view the $M$ branes on $\mathcal{C}$ as a probe. In this context, integrating out the gauge fields $A$ will leave us with an effective theory on the probe brane, which is an $S U(M)$ Chern-Simons on $\mathcal{C}$ plus some corrections. Let us define

$$
\begin{aligned}
\exp (-F(t, V)) & =\frac{1}{\int[D A] \exp \left(-S_{C S}\left(A ; S^{3}\right)\right)} \int[D A] \exp \left[-S_{C S}\left(A ; S^{3}\right)+\sum_{n=1}^{\infty} \frac{1}{n} \operatorname{tr} U^{n} \operatorname{tr} V^{-n}\right] \\
& =\langle Z(U, V)\rangle_{S^{3}}
\end{aligned}
$$

which can be viewed as the generating functional for all the observables of the ChernSimons gauge theory on $S^{3}$ associated to the unknot. Then we obtain an effective theory on the $\mathcal{C}$ brane, which is the deformation of the Chern-Simons theory as

$$
S=S_{C S}(\tilde{A} ; \mathcal{C})+F(t, V)
$$

Here $S_{C S}(\tilde{A} ; \mathcal{C})$ deonotes the Chern-Simons action on $\mathcal{C}$.

\subsection{Evaluation}

Let us evaluate $\langle Z(U, V)\rangle_{S^{3}}$ explicitly when the loop $q(s)$ is the unknot, i.e. a simple

circle in $S^{3}$ which represents a trivial knot. In this case it is known [11] that, for an admissible representation $\mathcal{R}_{j}$ of $S U(N)$, the Wilson loop expectation value is

$$
\left\langle\operatorname{Tr}_{\mathcal{R}_{j}} U\right\rangle_{S^{3}}=\frac{S_{0 j}}{S_{00}}
$$


where $S_{i j}$ is the modular transformation matrix for the characters of the $S U(N)$ current algebra at level $k$. If we know $\left\langle\operatorname{Tr}_{\mathcal{R}_{j}} U\right\rangle$ for all the representations, we can compute any product of traces of $U^{n}$ in the fundamental representation by using the Frobenius relation,

$$
\operatorname{tr} U^{n_{1}} \cdots \operatorname{tr} U^{n_{h}}=\sum_{Y} \chi\left(Y ;, n_{1}, \ldots, n_{h}\right) \operatorname{Tr}_{R(Y)} U
$$

where $Y$ is the Young tableau with $n=n_{1}+\cdots+n_{h}$ boxes, $R(Y)$ is the corresponding representation of $S U(N)$, and $\chi\left(Y ; n_{1}, \cdots, n_{h}\right)$ is the character of the representation of the permutation group $\mathcal{S}_{n}$ corresponding to $Y$, evaluated for the permutation with cycles of sizes $n_{1}, \cdots, n_{h}$ (for example, see [12]). To actually evaluate $\left\langle\operatorname{tr} U^{n_{1}} \cdots \operatorname{tr} U^{n_{h}}\right\rangle_{S^{3}}$, we use the following trick. We first note that $S_{0 j}$ is given by

$$
S_{0 j}=\sum_{w \in \mathcal{W}} \epsilon(w) \exp \left[-\frac{2 \pi i}{k+N}\left(w(\rho), \lambda_{j}+\rho\right)\right],
$$

where $\mathcal{W}$ is the finite Weyl group of $S U(N), \epsilon(w)= \pm 1$ is the parity of the element $w \in \mathcal{W}$ $\lambda_{j}$ is the weight vector for the representation $j$, and $\rho$ is a half of the sum of positive roots. Therefore $S_{0 j} / S_{00}$ takes the form of the character of the finite dimensional group $S U(N)$, namely

$$
\left\langle\operatorname{Tr}_{\mathcal{R}_{j}} U\right\rangle_{S^{3}}=\operatorname{Tr}_{\mathcal{R}_{j}} U_{0},
$$

where $U_{0}$ is a fixed element of $S U(N)$ which, in the fundamental representation, takes the form

$$
U_{0}=\left(\begin{array}{ccccc}
e^{\frac{\pi i(N-1)}{k+N}} & 0 & 0 & \cdots & 0 \\
0 & e^{\frac{(N-3) \pi i}{k+N}} & 0 & \cdots & 0 \\
0 & 0 & \cdots & \cdots & 0 \\
0 & 0 & 0 & \cdots & e^{\frac{\pi i(1-N)}{k+N}}
\end{array}\right) .
$$

Since $U_{0}$ in (3.10) is the same for any $\mathcal{R}_{j}$, to evaluate correlation functions of the Wilson loops, we can simply replace $U$ by the $c$-number matrix $U_{0}$ as

$$
\left\langle\operatorname{tr} U^{n_{1}} \operatorname{tr} U^{n_{2}} \cdots \operatorname{tr} U^{n_{h}}\right\rangle=\operatorname{tr} U_{0}^{n_{1}} \operatorname{tr} U_{0}^{n_{2}} \cdots \operatorname{tr} U_{0}^{n_{h}},
$$

and

$$
\operatorname{tr} U_{0}^{n}=\frac{\sin \left(\frac{n N \pi}{k+N}\right)}{\sin \left(\frac{n \pi}{k+N}\right)}=-i \frac{e^{n t / 2}-e^{-n t / 2}}{2 \sin (n \lambda / 2)},
$$

where $\lambda$ and $t$ are as defined in (2.1). Substituting this back into (3.4), we find

$$
\langle Z(U, V)\rangle_{S^{3}}=\exp \left[-i \sum_{n=1}^{\infty} \frac{e^{n t / 2}-e^{-n t / 2}}{2 n \sin (n \lambda / 2)} \operatorname{tr} V^{-n}\right] .
$$

As we will see, this is exactly the form we expect for the topological string on the resolved conifold geometry. 


\subsection{Conifold transition of the Wilson loop}

In the case of the unknot, it is straightforward to identify the effect of the conifold transition on the Lagrangian submanifold $\mathcal{C}$. Let us start with $T^{*} S^{3}$ expressed as (2.10), and consider the following anti-holomorphic involution on it.

$$
y_{1,2}=\bar{y}_{1,2}, \quad y_{3,4}=-\bar{y}_{3,4} .
$$

Since the symplectic form $\omega$ changes its sign under the involution, its fixed point set is automatically a Lagrangian submanifold of $T^{*} S^{3}$. This will be our $\mathcal{C}$. If we write $y_{\mu}=x^{\mu}+i p_{\mu}$, the invariant locus of the action (3.15) is

$$
p_{1,2}=0, \quad x^{3,4}=0
$$

and the equation (2.10) becomes

$$
\left(x^{1}\right)^{2}+\left(x^{2}\right)^{2}=a^{2}+\left(p_{3}\right)^{2}+\left(p_{4}\right)^{2} .
$$

Therefore $\mathcal{C}$ intersects with $S^{3}$ along the equator of $S^{3}$, i.e. the loop $q(s)$ is the unknot. The loop $q(s)$ in this case is identified with

$$
q(s): \quad\left(x^{1}\right)^{2}+\left(x^{2}\right)^{2}=a^{2}, \quad x^{3}=x^{4}=0 .
$$

To define $\mathcal{C}$ after the conifold transition, we continue to identify it with the invariant locus of the anti-holomorphic involution. To describe this explicitly let us use the coordinates $(u, v, z)$ or $\left(\tilde{u}, \tilde{v}, z^{-1}\right)$ defined by (2.13) and (2.15). In these coordinates, the $Z_{2}$ invariant set $\mathcal{C}$ is characterized by

$$
u=\overline{\tilde{v}}, \quad v=-\overline{\tilde{u}},
$$

and the conifold equation (2.14) restricted on $\mathcal{C}$ becomes

$$
u \bar{u}=v \bar{v} .
$$

The complex coordinate on the base $S^{2}$ defined by (2.15) is

$$
z=-\frac{u}{\bar{v}}
$$


Because of (3.19), $z$ is pure phase. Therefore one may view that $\mathcal{C}$ is a line bundle over the equator $|z|=1$ of $S^{2}$ (the fiber being the subspace of $\mathcal{O}(-1)+\mathcal{O}(-1)$ given by $u+z \bar{v}=0)$. In particular, $\mathcal{C}$ intersects with the base $S^{2}$ along $|z|=1$. See Figure 3. Since the intersection is one-dimensional, $\mathcal{C}$ remains a Lagrangian submanifold even after the $S^{2}$ is blown up and the symplectic form $\omega$ is modified.

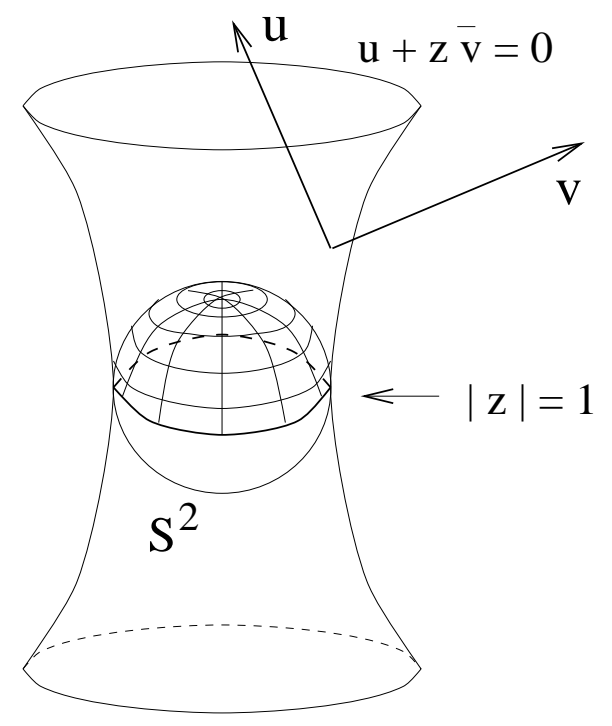

Figure 3: After the conifold transition, the Lagrangian 3-cycle touches the base $S^{2}$ along the equator $|z|=1$ and extends in the fiber directions following the constraint $u+z \bar{v}=0$. The worldsheet instanton can either wrap the northern hemisphere, as shown in the figure, ending on the equator, or wrap the southern hemisphere.

According to the conjecture of [3], topological string with $N$ D-branes wrapping on the base $S^{3}$ of $T^{*} S^{3}$ is equivalent to topological string on the resolved conifold without D-branes. Here we are adding $M$ D-branes on $\mathcal{C}$ on one side, and have traced it over to the other side. On the $T^{*} S^{3}$ side, the effective theory on the $M$ probe branes was the ChernSimons action plus some corrections (3.6). So the conjecture gives the falsifiable prediction that, after the conifold transition, we should also see the effective theory on the brane to be a deformed version of the Chern-Simons theory (3.6). Indeed it has been shown in [4 that when there are holomorphic maps from Riemann surfaces with boundaries to the target space, with boundaries lying on the D-brane, the Chern-Simons action gets deformed. In the original geometry of $T^{*} S^{3}$, there are no such maps. However we got the deformation by integrating the gauge theory on $S^{3}$ and the scalar field living on the knot. At large $N$, we have made a transition to a new geometry without any other sectors. But now, there are non-trivial holomorphic maps that can end on $\mathcal{C}$ ! Since $\mathcal{C}$ intersects with the base $S^{2}$ 
of the resolved conifold along $|z|=1$, there are holomorphic maps from Riemann surface with a boundary with the image having the topology of disc. It is shown in [4] that the effective theory on $\mathcal{C}$ should now be of the form as predicted in (3.6) with $F(t, V)$ given by

$$
F(t, V)=\sum_{g=0}^{\infty} \sum_{h=1}^{\infty} \sum_{n_{1}, \cdots, n_{h}}^{\infty} \lambda^{2 g-2+h} F_{g ; n_{1}, \cdots, n_{h}}(t) \operatorname{tr} V^{n_{1}} \cdots \operatorname{tr} V^{n_{h}}
$$

Here $F_{g ; n_{1}, \cdots, n_{h}}$ is the topological string amplitude on a genus- $g$ surface with $h$ boundaries. The factors $\operatorname{tr} V^{n_{i}}$ are picked up by the boundary of the worldsheet, which wraps $\left|n_{i}\right|$-times the equator of the $S^{2}$ either clockwise $n_{i}>0$ or counterclockwise $n_{i}<0$ depending on whether the worldsheet is mapped to the upper or the lower hemisphere.

To see that the Chern-Simons computation (3.14) agrees with this expectation, we note that, in the topological string computation, amplitudes are assumed to be analytic in $t$. By performing the analytic continuation司, we can rewrite $\langle Z(U, V)\rangle$ as

$$
\langle Z(U, V)\rangle_{S^{3}}=\exp \left[i \sum_{n=1}^{\infty} \frac{\operatorname{tr} V^{n}+\operatorname{tr} V^{-n}}{2 n \sin (n \lambda / 2)} e^{-n t / 2}\right]
$$

This agrees with the general form (3.21) expected for the topological string amplitude. We could make a more quantitative comparison by counting holomorphic maps. There are only two basic holomorphic maps (with the image being a disc) with boundaries on $\mathcal{C}$, which are the upper and the lower hemispheres of the $S^{2}$, together with their multicoverings, and with the higher genus coverings of them (see Figure 3). In particular, the comparison of (3.21) and (3.22) suggests that all the relevant instantons have one boundary ending on $\mathcal{C}$, wrapping the equator of $S^{2}$ either clockwise or counterclockwise. It would be interesting to verify the prediction of the Chern-Simons computation (3.22) explicitly using the worldsheet instanton calculus extending the results from the closed string case to open strings. In this paper, we will take an alternative route, by giving the target space interpretation of $F(t, V)$ and evaluate it explicitly by the Schwinger-type computation, similar to what was done in the closed string case in [10]. We will find that the prediction (3.22) is precisely reproduced in this way.

5 By the analytic continuation, we can replace $\sum_{n=1}^{\infty} n^{2 a} \operatorname{tr} V^{n} e^{n t / 2}$ by $-\sum_{n=1}^{\infty} n^{2 a} \operatorname{tr} V^{-n} e^{-n t / 2}+$ const for any integer $a$. 


\section{Target Space Interpretation}

Topological strings are useful in computing superpotential-type terms in the context of superstring compactification on Calabi-Yau manifold. In particular it is known [13] that topological closed strings amplitude $F_{g}\left(t_{i}\right)$ at genus $g$, where $t_{i}$ are the Kähler moduli of a Calabi-Yau threefold, computes the low energy effective theory terms of the Type IIA compactification,

$$
\int d^{4} x d^{4} \theta F_{g}\left(t_{i}\right)\left(W^{2}\right)^{g}
$$

where the integral is on the $4 d N=2$ superspace, $t_{i}$ denote a vector multiplet with the Kähler expectation values as the top element. $W_{\alpha \beta}$ denote the graviphoton multiplet (with self-dual graviphoton field strength as the top component), $\alpha, \beta$ denote symmetric spinor indices and

$$
W^{2}=W_{\alpha \beta} W_{\alpha^{\prime} \beta^{\prime}} \epsilon^{\alpha \alpha^{\prime}} \epsilon^{\beta \beta^{\prime}}
$$

In fact it was through this connection where $F_{g}\left(t_{i}\right)$ were reinterpreted in [10] in terms of spectrum of wrapped M2/D2 branes in the Calabi-Yau threefold. In particular it was shown that

$$
\sum_{g} F_{g}\left(t_{i}\right) \lambda^{2 g-2}=\sum_{n=1}^{\infty} \sum_{Q \in H_{2}(M, \mathbf{Z})} \sum_{s=0}^{\infty} N_{Q, s}(2 \sin (n \lambda / 2))^{2 s-2} \frac{e^{-n t_{Q}}}{n}
$$

where $t_{Q}=\int_{Q} k$ is the area of the cycle and $N_{Q, s}$ denotes the (net) number of $M_{2}$ brane bound states of charge $Q$ and $S U(2)_{L}$ content $\left[2(0)+\left(\frac{1}{2}\right)\right]^{\otimes s}$ (for more detail see [10]). This was obtained by computing the effective one-loop Schwinger-type correction to the terms of the form $R_{+}^{2} F_{+}^{2 g-2}$, with D2 brane bound states going around the loop [14]. The sum over $n$ above arises because every D2 brane can bind exactly once to an arbitrary number of D0 branes, i.e. every M2 bound state can have arbitrary momentum around the circle. In other words the sum over arbitrary number of D0 branes gives rise to a delta function, which effectively replaces the Schwinger time integral by a discrete sum represented by $n$ above. The factor of $(2 \sin (n \lambda / 2))^{2 s-2}$ arises from a $(2 \sin (n \lambda / 2))^{2 s}$ having to do with the extra contribution of a states of spin content $\left[2(0)+\left(\frac{1}{2}\right)\right]^{\otimes s}$ running around the loop in the Schwinger computation, as compared to a spin 0 which would give $(2 \sin (n \lambda / 2))^{-2}$.

We would like to repeat an analogous scenario for reinterpretation of topological $A$ model with D-branes which include a supersymmetric 3-cycle in the internal Calabi-Yau threefold as its worldvolume. There are various cases one can consider. We will consider in particular the Type IIA compactification on a Calabi-Yau, with one additional D4 brane 
wrapped around a supersymmetric 3-cycle $S$ and filling an $R^{2} \subset R^{4}$ in the uncompactified spacetime. Suppose the first Betti number of $S$ is $r=b_{1}(S)$. Then on $R^{2}$ subspace of $R^{4}$ live $r(2,2)$ supersymmetric chiral superfields $\Sigma^{i}$ corresponding to the scalar moduli of $S$ in the Calabi-Yau threefold [15]. The top component of this chiral field is a complex field whose phase is related to the expectation value of the Wilson line of the gauge field $A$ on the D4-brane around the corresponding 1-cycle of $S$. Moreover $\Sigma^{i}$ can be viewed as a $(2,2)$ vector multiplet on $R^{2}$. The $U(1)$ gauge field on $R^{2}$ corresponds to the magnetic 2-form $B$ field on the D4 brane $d B=* d A$ and taking the component of $B$ along the corresponding cycle in $S$, to yield a gauge field on $R^{2}$. One could generalize this by considering $M$ copies of the D4 brane. We will get in this case $M$ copies of the $U(1)$ gauge field and so the fields $\Sigma_{i}^{\alpha}$ will be naturally in the adjoint of $U(1)^{M}$. The permutation groups $\mathcal{S}_{M}$ which is the symmetry of D4 branes acts on this set of fields to permute the $\Sigma_{i}$. Giving vev to $\left\langle\Sigma_{i}^{\alpha}\right\rangle=\theta_{i}^{\alpha}$ allows us to think of each $i$-direction a diagonal $U(M)$ matrix of holonomy given by diagonal elements $\exp \left(i \theta_{i}^{\alpha}\right)$. Let us denote this $U(M)$ matrix by $V_{i}$.

Now we are ready to state what physical amplitude the topological string computes in the presence of D-branes. The topological strings in this case computes

$$
\int d^{4} x d^{4} \theta \delta^{2}(x) \delta^{2}(\theta) F_{g, h}\left(V_{i}, t\right)\left(W^{2}\right)^{g}(W \cdot v)^{h-1},
$$

where $W \cdot v=W_{\alpha \beta} v^{\mu \nu} \gamma_{\alpha \beta}^{\mu \nu}$, and $v^{\mu \nu}$ denotes the vector orthogonal to the noncompact worldvolume of D4 brane, and $\gamma^{\mu \nu}$ are the usual gamma matrices. The delta function above localizes the contribution to the superspace defined by the noncompact part of the D4 brane. Here

$$
F_{g, h}\left(V_{i}, t\right)=\sum_{n_{j}^{i}} F_{g, n_{\alpha}^{i}}(t) \prod_{\alpha=1}^{h} \operatorname{tr} \otimes_{i=1}^{b_{1}(S)} V_{i}^{n_{\alpha}^{i}},
$$

and $F_{g, n_{\alpha}^{i}}(t)$ denotes the topological string amplitude at genus $g$ with $h$ holes, labeled by $\alpha=1, \cdots, h$ and where on each hole $\alpha$ the circle on the Riemann surface is mapped to the boundary of $S$ characterized by the $H_{1}(S)$ class $n_{\alpha}^{i}$. The trace factors above are just the usual Chan-Paton factors. The derivation of (4.2) is similar to that for the closed string case and can be done most conveniently in the Berkovits formalism [16], similar to what was done in the closed string case for the Calabi-Yau topological amplitudes in [17].

As in the closed string case, we would like to connect (4.2) with contributions due to wrapped D2 branes. The main additional ingredient in this case is that the D2 brane can end on the D4 brane $S$. This will give a state magnetically charged under the $U(1)^{M}$ 
living on the D4 brane. One term included in (4.2) after doing the superspace integral is a term of the form $R F^{2 g-2+h}$ where $F$ denotes the expectation value of the $4 d$ graviphoton field strength restricted to the uncompactified worldvolume of the $\mathrm{D} 4$ brane. If we give a vev to the graviphoton, $\langle F\rangle=\lambda$, this would compute correction to $\int R$ as a function of

$$
F\left(t, V_{i}\right)=\sum_{g, h} F_{g, h}\left(t, V_{i}\right) \lambda^{2 g-2+h} .
$$

This is the summed up version of the topological string amplitudes over all genera and holes, where the role of the string coupling constant $\lambda$ is played by the vev of $F$.

We thus compute the contribution of magnetically charged D4 branes ending on $S$ to $\int R$ in the presence of the background $F$. Each such particle will transform according to some representation $\mathcal{R}$ for $\otimes_{i} U_{i}(1)^{M} / \mathcal{S}_{M}$, where $i$ runs from $i=1, \cdots, b_{1}(S)$. We in principle do not know if they form representation of $U(M)$ (for each element of $b_{1}(S)$ ) but nevertheless we can assign them to representatitions of $U(M)$ if we allow negative multiplicity. This is because any $\mathcal{S}_{M}$ symmetric spectrum for $U(1)^{M}$ can be written as combination of weights appearing in various representations of $U(M)$. From this point on, we will therefore take $\mathcal{R}$ to be a representation of $U(M)$ (for each $b_{1}(S)$ ) and allow negative multiplicities. In addition every such state is characterized by its bulk D2 brane charge $Q \in H_{2}(M, S)$, i.e. a 2-cycle in the Calabi-Yau threefold ending on $S$. Every such field will be represented by some spin $s$ field in 2 dimensions, where $2 s$ is a positive or negative integer. To determine $s$, it is most convenient to view it from the M-theory perspective; In the strong coupling limit this geometry gets related to M-theory on the Calabi-Yau threefold, with M5 brane filling $S \times R^{3}$. The magnetic charged state correspond to particles in 3 dimensions with $M 2$ branes ending on the M5 brane. The little group of massive particles in three dimensions is $S O(2)$ and so the particle carries a $3 d$ spin $s$. Upon reduction to $2 d$, this particle is realized by a field with spin $s$. Moreover each such particle can be bound to an arbitrary number of D0 branes. This is clear also from the M-theory perspective as each particle carries an arbitrary momentum as we go down from 3 dimensions to 2 on a circle. The computation then is as in the closed string case, where we effectively get the Schwinger computation for a scalar field (with the supersymmetry being responsible for generating $\int R$ ), and

$$
F\left(t, V_{i}\right)=i \sum_{n=-\infty}^{\infty} \sum_{\mathcal{R}, Q, s} \int_{0^{+}}^{\infty} \frac{d \tau}{\tau} \frac{N_{\mathcal{R}, Q, s}}{2 \sin (\tau \lambda / 2)} e^{i s \lambda \tau} \operatorname{Tr} e^{\left(-m_{R, Q}+2 \pi i n\right) \tau}
$$

6 There is a priori no reason why $M$ coincident branes give rise to a magnetic $U(M)$ gauge theory. 
Here $N_{\mathcal{R}, Q, s}$ denotes the net number of magnetically charged states with charges given by $\mathcal{R}, Q$ and spin $s$. The parameter $\tau$ is the Schwinger time, the sum over $n$ is the sum over the D0 brane bound states, and $m_{R, Q}+2 \pi i n$ denotes the BPS mass of the wrapped D2 brane, which is given by

$$
\operatorname{Tr} e^{-m_{R, Q}+2 \pi i n}=e^{-t_{Q}+2 \pi i n} \operatorname{Tr}_{\mathcal{R}} \prod_{i=1}^{b_{1}(S)} V_{i},
$$

where $t_{Q}=\int_{Q} k$. To see how the above expression arises, note that for one D4 brane this follows from the fact that $\int_{Q} k$ is just the bulk contribution to the BPS formula and $\operatorname{Tr}_{\mathcal{R}} \prod_{i=1}^{b_{1}(S)} V_{i}$ arises from the fact that giving vev to the $U(1)$ fields for each one gives a BPS mass $q \theta$, where $q$ is the charge under $U(1)$ and $\theta$ denotes the vev of a the scalar in $U(1)$ multiplet. Doing the sum over $n$ in the above gives a delta function $\sum_{n=-\infty}^{\infty} \delta(\tau-n)$, which converts the $\tau$ integral into a sum, and we obtain

$$
F\left(t, V_{i}\right)=i \sum_{n=1}^{\infty} \sum_{\mathcal{R}, Q, s} \frac{N_{\mathcal{R}, Q, s}}{2 n \sin (n \lambda / 2)} e^{n\left(-t_{Q}+i s \lambda\right)} \operatorname{Tr}_{\mathcal{R}} \prod_{i=1}^{b_{1}(S)} V_{i}^{n} .
$$

Note that to compare it with (4.3) one has to expand the trace from representation $R$ in terms of fundamental representation of $U(M)$. Note that the above expression has strong integrality predictions which would be interesting to verify.

Note that for the special case of $g=0, h=1$, i.e. the disc amplitude (4.2) computes theta terms in gauge theory. Namely for each diagonal element of $V_{i}$, denoted by $\exp \left(i \theta_{i}^{\alpha}\right)$ the term

$$
\frac{\partial F_{0,1}}{\partial \theta_{i}^{\alpha}}\left(t, V_{i}\right)
$$

denotes the correction to the theta term $\int F_{i}^{\alpha}$ where $F_{i}^{\alpha}$ denotes the field strength for the corresponding $U(1)$ gauge field in $2 d$. From (4.4) we can read the prediction for this, which is given by

$$
\begin{aligned}
& i \sum_{n=1}^{\infty} \sum_{v_{i}^{\alpha} \in \mathcal{R}, Q, s} N_{\mathcal{R}, Q, s} q_{i}^{\alpha} \frac{1}{n} e^{-n\left(t_{Q}+i v_{i}^{\alpha} \theta_{i}^{\alpha}\right)} \\
& =-i \sum_{v_{i}^{\alpha} \in \mathcal{R}, Q, s} N_{\mathcal{R}, Q, s} q_{i}^{\alpha} \log \left(1-e^{-t_{Q}-i v_{i}^{\alpha} \theta_{i}^{\alpha}}\right) \\
& =-i \sum_{m=-\infty}^{\infty} \sum_{v_{i}^{\alpha} \in \mathcal{R}, Q, s} N_{\mathcal{R}, Q, s} q_{i}^{\alpha} \log \left(t_{Q}+i v \cdot \theta+2 \pi i m\right)
\end{aligned}
$$


which is the expected correction to the theta angle in $2 d N=2$ gauge theory from charged matters with BPS masses $t_{Q}+i v \cdot \theta+2 \pi i m$ and charge $q_{i}^{\alpha}$ (see in particular a similar correction which was studied in [18]). Note that from (4.4) we can write $F_{0,1}$ in the form

$$
F_{0,1}\left(t, V_{i}\right)=i \sum_{n=1}^{\infty} \sum_{v \in \mathcal{R}, Q, s} N_{\mathcal{R}, Q, s} \frac{1}{n^{2}} e^{-n\left(t_{Q}+i v \cdot \theta\right)}
$$

\subsection{Applications to $N=1, d=4$ systems}

In the above to interpret the topological string amplitudes with boundaries, we used a D4 brane system with worldvolume $R^{2} \times S$. Instead we could have used a D6 brane system with worldvolume $R^{4} \times S$. This would only make sense in the context of noncompact Calabi-Yau manifolds (otherwise the flux of D6 brane charge has nowhere to go). Then again the fields $V_{i}$ correspond to chiral fields in $d=4$. In this case the interpretation of the topological amplitudes given in (4.2) gets modified. The simplest case to consider turns out to be $h=1$ and $g$ arbitrary. In this case the topological string amplitudes compute

$$
\int d^{4} x d^{4} \theta \delta^{2}(\theta) F_{g, 1}\left(V_{i}, t\right)\left(W^{2}\right)^{g}
$$

and in particular $F_{0,1}$, i.e. topological disc amplitudes computes superpotential terms for $\mathrm{N}=1$ theories in four dimensions. This has already been noted in [13] [19] and is being further studied in [20]. Let us call $F_{0,1}=W\left(V_{i}, t\right)$, the superpotential. From the formula (4.5) we thus have a general expression for the superpotential $W$ in terms of the spectrum of BPS states namely

$$
W\left(t, V_{i}\right)=\sum_{n=1}^{\infty} \sum_{v \in \mathcal{R}, Q, s} N_{\mathcal{R}, Q, s} \frac{1}{n^{2}} e^{-n\left(t_{Q}+i v \cdot \theta\right)} .
$$

A special simple case of this is when we have a single brane where $V_{i}$ can be viewed as a complex superfield $e^{i \theta_{i}}$. Given that our derivation of this term seems to require 2-dimensional concepts, it is natural to ask if we could also reproduce this from a 4 dimensional viewpoint. As we will see this is also possible. In the case of D6 branes with worldvolume $R^{4} \times S$, the magnetically charge branes are D4 branes ending on the D6 brane. This will correspond to a domain wall in $R^{4}$. The expression (4.7) then suggests that we should be able to relate the superpotential term, to the structure of domain walls

7 As before we are dropping terms polynomial in $t$ and $\theta$ 's which would have corresponded to $n=0$ in the above sum. 
by "integrating them out." However unlike the 2-dimensional case, we cannot send the domain walls around the loop, so the question is how would we obtain such an expression by integrating fields out in the $4 d$ case.

A hint comes from the recent work [21] and a similar case studied in [22], where it was shown how extra fields are relevant for reproducing the domain wall structure. For each domain wall, we introduce a field $Y_{\alpha}$ as a chiral superfield, which characterizes it by shifting by $2 \pi i$ as we go across the domain wall. Since we can have a priori an arbitrary number of domain walls, we must thus have infinitely many vacua, given by shifting the expectation value of $Y_{\alpha}$ by $2 \pi i n$. Moreover the tension for the domain wall should be given by the BPS formula,

$$
W(Y+2 \pi i)-W(Y)=2 \pi i Z
$$

The superpotential satisfying these constraints which was found in [21] in a similar context is given by

$$
W=Z_{\alpha} Y_{\alpha}+\exp \left(-Y_{\alpha}\right) .
$$

Note that the critical points obeying $d_{Y} W=0$ are given by

$$
\exp \left(-Y_{\alpha}\right)=Z_{\alpha}
$$

namely $Y_{\alpha}=-\log Z_{\alpha}+2 \pi i n$, and that the equation (4.8) is satisfied. If we integrate out the hidden variable $Y_{\alpha}$ we obtain by replacing $Y_{\alpha}$ by its critical value a superpotential term

$$
W=Z_{\alpha}\left(1-\log Z_{\alpha}\right) .
$$

In the case at hand for each element $v$ in a representation $v \in \mathcal{R}$ of the magnetic charges, and charge $Q$ in the bulk we have $N_{\mathcal{R}, Q, 0}$ net BPS domain walls for each integer m, with BPS tension

$$
Z_{\alpha}=t_{Q}+i v \cdot \theta+2 \pi i m
$$

Plugging this into (4.9) and summing over all such states, we obtain the formula (4.7). 


\subsection{Application to D-brane on $\mathcal{O}(-1)+\mathcal{O}(-1)$ over $S^{2}$}

In this section we will show how the results of the previous section are in agreement with the above analysis, and in particular gives an independent derivation for topological $A$-model in $\mathcal{O}(-1)+\mathcal{O}(-1)$ over $S^{2}$ with the 3 cycle $\mathcal{C}$ we have discussed. In that case the

$b_{1}(\mathcal{C})=1$ and so we have only one chiral field, which gives rise to one holonomy matrix $V$. It is clear what the magnetically charges states are; they correspond to the D2 brane wrapping the northern hemisphere and ending on $S$ or the one wrapping the southern hemisphere and ending on $S$. The first one has $\left(t_{Q}, \mathcal{R}\right)$ given by $(t / 2$, fundamental) and the second has $(t / 2$, anti-fundamental). They both have spin 0 , as there is no moduli for them. We thus obtain from (4.4):

$$
F(t, V)=i \sum_{n=1}^{\infty} \frac{\operatorname{tr} V^{n}+\operatorname{tr} V^{-n}}{2 n \sin (n \lambda / 2)} e^{-n t / 2},
$$

which agrees with the knot invariant predicted for the unknot, as discussed in the previous section, with $t=2 \pi i N /(k+N)$ and $\lambda=2 \pi /(k+N)$.

\section{Suggestions for Future Work}

Here we have mainly concentrated in computing the expectation value of the Wilson loop for a simple knot, and have found a striking agreement with the predictions of topological strings in the Large $N$ limit, anticipated from the large $N$ Chern-Simons/topological string duality proposed in [3]. This was done by independently computing both sides and checking that they agree. On the topological string we used D-branes ending on branes to get a prediction for what the topological string should reduce to.

It would be nice to generalize this for arbitrary knots. There are two obstacles to overcome. On the Chern-Simons side we need to compute

$$
\langle Z(U, V)\rangle_{S^{3}}=\left\langle\exp \left[\sum_{n=1}^{\infty} \operatorname{tr} \frac{1}{n} U^{n} \operatorname{tr} V^{-n}\right]\right\rangle_{S^{3}} .
$$

This is already rather difficult to do, even though in principle it should be possible. The reason for this is the appearance of all the powers of $\operatorname{tr} U^{n}$. In particular we need to know all correlations $\left\langle\operatorname{tr} U^{n_{1}} \cdots \operatorname{tr} U^{n_{k}}\right\rangle$. For a general knot, the correlators do not decouple, unlike the unknot (3.12). Even though it is in principle possible to compute them, they have not been computed in the full generality we need. Nevertheless the structure of the 
answer for the $\langle\operatorname{tr} U \cdots \operatorname{tr} U\rangle$ dictated by the Skein relations [11] are compatible with the general answer expected for the knot invariants, which follows from the discussion in the previous section, in particular (4.4). Note that we are mapping all the knot invariants for arbitrary representations, into new integer invariants $N_{Q, \mathcal{R}, s}$, where two different $Q$ 's differ by an integer (so they can be parametrized by an integer), $s$ denotes a positive (or zero) spin representation, and $\mathcal{R}$ is a representation of $U(M)$ for any $M$. We expect that for each knot $\mathcal{R}$ will stabilize for large enough $M$. What we mean by this is that it will given by representations with finite number of boxes in the Young tableau (or whose conjugate has finite number of boxes). Thus for $M$ large enough we have 'probed' the full content of $\mathcal{R}$ representation (for example for the unknot we found that already $M=1$ is sufficient and the structure for higher $M$ 's can be induced from that). This may be a very useful reformulation of knot invariants, somewhat analogous to the reformulation of Gromov-Witten invariants, in terms of the new invariants defined in [10]. In particular the knot invariants would be given by 8

$$
\langle Z(U, V)\rangle_{S^{3}}=\exp \left[i \sum_{n=1}^{\infty} \sum_{\mathcal{R}, Q, s} \frac{N_{\mathcal{R}, Q, s}}{2 n \sin (n \lambda / 2)} e^{n\left(-t_{Q}+i s \lambda\right)} \operatorname{Tr}_{\mathcal{R}} V^{n}\right]
$$

For understanding this new formulation of knot invariants, we also have to construct a Largrangian submanifold for an arbitrary knot, on the resolution of the conifold, generalizing our explicit construction for the unknot. That there should be such a canonical Lagrangian submanifold for each knot is natural. This is because we already have identified, for an arbitrary knot, the Lagrangian submanifold on the $T^{*} S^{3}$ side, and small resolution does not change the geometry of the Lagrangian submanifold at infinity. So with some deformation near the origin we should be able to obtain the Lagrangian submanifold after the conifold singularity is blown up. Then we are predicting that the topological string amplitudes, whose answer must have the structure (4.4), compute the knot invariants. This would be a very important subject to develop, not only for a deeper understanding of knot invariants, but also for a better understanding of topological strings with boundaries.

8 In comparing with the knot invariants, it is natural to do analytic continutation, as we have seen in the case of the unknot, in the case where the representation are tensor products of the fundamental representation of $U(M)$ (as opposed to tensor products of the anti-fundamental representation). This would be equivalent to replacing for those representations the corresponding $t_{Q} \rightarrow-t_{Q}$, and changing the sign of the power of $V$ and putting an overall sign in front of those terms. 
Also we have seen in this paper how we can compute superpotential terms on the Type IIA compactifications on Calabi-Yau threefold in the presence of D6 brane, at least in some simple cases. In a more general case, doing the computation on the mirror should be simpler [23]. Some examples of this have been recently studied in [20]. This may well lead to a method for geometric engineering of $N=1$ and its solution in terms of the type IIB mirror. Namely, we start with the usual geometric engineering of $N=2$, introduce additional D6 branes to break the $N=2$ to $N=1$ (effectively giving mass terms to the adjoint fields) and then using the type IIB mirror to compute the superpotential terms generated, very much the way prepotential for $N=2$ theories were computed using mirror symmetry. This would be very exciting to develop further.

\section{Acknowledgements:}

We are grateful to N. Berkovits, R. Gopakumar, K. Hori, S. Sinha, C. Taubes and T. Taylor for valuable discussions. H.O. would like to thank the theory group of Harvard University, where this work was initiated and completed.

The research of H.O. was supported in part by NSF grant PHY-95-14797, DOE grant DE-AC03-76SF00098, and the Caltech Discovery Fund. The research of C.V. is supported by NSF Grant No. PHY-9218167.

\section{Note Added}

After the completion of this work, beautiful computations were done [24] for checking the predictions made in this paper for the case of torus knots and finding impressive agreement with what was anticipated. We would like to thank J. Labastida and M. Mariño, who informed us of their computation prior to publication which prompted us to correct an error we had made in the Schwinger computation in an earlier version of this paper. 


\section{References}

[1] V. Periwal, "Topological Closed-string Interpretation of Chern-Simons Theory," Phys. Rev. Lett. 71 (1993) 1295.

[2] M.R. Douglas, "Chern-Simons-Witten Theory as a Topological Fermi Liquid," hepth/9403119.

[3] R. Gopakumar and C. Vafa, "On the Gauge Theory/Geometry Correspondence," hep-th/9811131.

[4] E. Witten, "Chern-Simons Gauge Theory as a String Theory," hep-th/9207094.

[5] C. Faber and R. Pandharipande, "Hodge Integrals and Gromov-Witten Theory," math.AG/9810173.

[6] P. Candelas, X.C. De La Ossa, P. S. Green and L. Parkes, "A Pair of Calabi-Yau Manifolds as an Exactly Soluble Superconformal Theory," Nucl. Phys. B359 (1991) 21.

[7] S. Hosono, A. Klemm, S. Theisen and S.-T. Yau, "Mirror Symmetry, Mirror Map and Applications to Complete Intersection Calabi-Yau Spaces," Nucl. Phys. B433 (1995) 501; hep-th/9406055.

[8] M. Bershadsky, S. Cecotti, H. Ooguri and C. Vafa, "Holomorphic Anomalies in Topological Field Theories," Nucl. Phys. B405 (1993) 279; hep-th/9302103.

[9] M. Bershadsky, S. Cecotti, H. Ooguri and C. Vafa, "Kodaira-Spencer Theory of Gravity and Exact Results for Quantum String Theory," Commun. Math. Phys. 165 (1994) 311 ; hep-th/9309140.

[10] R. Gopakumar and C. Vafa, "M-Theory and Topological Strings I, II," hepth/9809197, 9812127.

[11] E. Witten, "Quantum Field Theory and the Jones Polynomial," Commun. Math. Phys. 121 (1989) 351.

[12] D.J. Gross and W. Taylor, "Twists and Wilson Loops in the String Theory of TwoDimensional QCD," Nucl. Phys. B403 (1993) 395; hep-th/9303026.

[13] I. Antoniadis, E. Gava, K. S. Narain and T. R. Taylor, "Topological Amplitudes in Superstring Theory," Nucl. Phys. B413 (1994) 162; hep-th/9307158.

[14] I. Antoniadis, E. Gava, K. S. Narain and T. R. Taylor, " $N=2$ Type II - Heterotic Duality and Higher Derivative F-Terms," Nucl. Phys. B455 (1995) 109; hep-th/9507115.

[15] A. Strominger, S.-T. Yau and E. Zaslow, "Mirror Symmetry is T Duality," Nucl. Phys. B479 (1996) 243; hep-th/9606040.

[16] N. Berkovits, private communication.

[17] N. Berkovits and C. Vafa, " $N=4$ Topological Strings," Nucl. Phys. B433 (1995) 123; hep-th/9407190.

[18] A. Hanany and K. Hori, "Branes and $N=2$ Theories in Two Dimensions," Nucl. Phys. B513 (1998) 119; hep-th/9707192. 
[19] I. Brunner, M. R. Douglas, A. Lawrence and C. Romelsberger, "D-Branes on the Quintic," hep-th/9906200.

[20] S. Kachru, S. Katz, A. Lawrence, and J. McGreevy, "Open String Instantons and Superpotentials," to appear.

[21] K. Hori and C. Vafa, "Mirror Symmetry," to appear.

[22] S. Gukov, C. Vafa and E. Witten, "CFT's from Calabi-Yau Four-folds," hepth/990670.

[23] C. Vafa, "Extending Mirror Conjecture to Calabi-Yau with Bundles," hep-th/9804131.

[24] J. Labastida and M. Mariño, "Polynomial Invariants for Torus Knots and Topological Strings," to appear. 